\title{
CONSTRUCCIÓN EN RED DE UN CURRÍCULO BASADO EN COMPETENCIAS
}

\section{RED CONSTRUCTION OF A COMPETENCY BASED CURRICULUM}

\author{
Ana María Vásquez Aqueveque * \\ Rosa Apablaza Correa ** \\ LIDIA OSORIO OLIVARES ${ }^{* * *}$ \\ Josefina ZúÑiga Aguirre ${ }^{* * * *}$
}

\begin{abstract}
RESUMEN
La diversidad y el aumento de instituciones de educación terciaria, junto con el incremento de oferta de carreras, posibilita el acceso a la educación superior a segmentos de la población hasta ahora excluidos, esto se traduce en un nuevo perfil de estudiante que se inserta en la educación terciaria. Asimismo, la reforma en salud, los cambios demográficos, epidemiológicos, sociales, culturales, económicos, el avance sostenido de enfermería como profesión y disciplina, exigen un replanteamiento en la formación y proponer un perfil de egreso acorde a las realidades y a las proyecciones enunciadas. Conscientes de esta situación se diseñó un currículo basado en competencias, centrado en el estudiante, que posibilite movilidad estudiantil. Para realizar el diseño precedentemente indicado se estableció un convenio de colaboración entre las cuatro instituciones de la red, se contrató a expertos que orientaron el diseño curricular, acorde a las tendencias en la disciplina de enfermería y educación. Se visitaron diferentes países para recabar experiencia; se analizaron documentos, se realizaron talleres con participación de diversos estamentos, donde se consideraron las competencias que se requieren en el ámbito laboral y concordar el perfil de egreso. Como productos se elaboró un marco contextual, con una amplia mirada, donde se relacionan los principales conceptos que iluminan la propuesta curricular, se definió un perfil y las competencias de egreso, se elaboró el plan de estudio asignando créditos a las asignaturas acorde al sistema de crédito transferible propuesto por los vicerrectores de las universidades adscritas al Consejo de Rectores de Chile.
\end{abstract}

Palabras clave: Currículum, educación basada en competencias, educación en enfermería.

\begin{abstract}
The diversity and the increase of tertiary education institutions, as well as increasing career offer, allows access to higher education to segments of the population hitherto excluded, resulting in a new student profile that is inserted into tertiary education. Furthermore, health reform, demographic, epidemiological, social, cultural, economic changes, and sustained progress of nursing as a profession and discipline, requires a rethinking in the formation and propose a profile of graduates according to the reality and the contained projections. Aware of this situation a competency-based curriculum, student-centered, which enables student mobility was designed.

To make the design previously indicated, a collaboration agreement among the four institutions in the network, was established. Experts that guided the curriculum, according to trends in the discipline of nursing, and education, were hired. Different countries were visited to gather experience, documents were analyzed, as well as workshops with the participation of various branches, which considered the skills required

\footnotetext{
*Enfermera, académica Departamento de Enfermería Universidad de La Serena, Chile. Email: amvas@userena.cl

** Enfermera, académica Departamento de Enfermería de la Universidad de Antofagasta, Chile. Email: rapablaza@uantof.cl

*** Enfermera, académica Escuela de Enfermería Universidad Arturo Prat, Chile. Email: losorio@unap.cl

${ }^{* * * *}$ Enfermera, académica Carrera de Enfermería Universidad de Tarapacá, Chile. Email: jzuniga@uta.cl
} 
at workplace and match the graduate profile as well. As products, a contextual framework was developed, with a broad glance, where the main concepts that illuminate the curriculum proposal relate themselves; a profile and graduate competencies were defined and the curriculum assigning credits to subjects according to the transferable credit system proposed by the vice chancellors of the universities attached to the Council of Rectors of Chile, was prepared.

Key words: Curriculum, competency-based education, education, nursing.

Fecha recepción: 07/09/11 Fecha aceptación: 29/12/11

\section{INTRODUCCIÓN}

El desarrollo de enfermería como disciplina ha experimentado avances importantes en las últimas décadas, lo que ha permitido perfilar, con mayor claridad, su objeto de estudio y la identidad profesional (el cuidado de las personas). En este contexto, la formación profesional de enfermería ha experimentado modificaciones orientadas, fundamentalmente, a desarrollar en los estudiantes competencias eficaces que les permitan, en la vida profesional, un desempeño acorde a una realidad que exige capacidad de adaptación, flexibilidad, interés en la actualización. Sin embargo, esta adaptación no es suficiente, pues no se han logrado cambios que aseguren efectivamente un mejor posicionamiento del profesional de enfermería en el ámbito social y laboral, y una mayor pertinencia en su formación, en torno al cuidado, como ciencia humana y motivación al aprendizaje continuo, para toda la vida.

En Europa, desde el año 2001, la disciplina de Enfermería está participando en el Proyecto Tuning II, que ha convocado a trece países de la Comunidad Económica Europea (175 universidades) con el propósito de desarrollar conjuntamente perfiles profesionales (competencias), y establecer resultados de aprendizaje, intercambiar información sobre los currículos, asociar otras áreas temáticas, construir vínculos entre las redes de universidades y otras instancias cualificadas, coordinarse con todos los beneficiarios relevantes, de tal manera de crear una base de compa- rabilidad y transparencia, facilitar la movilidad y convergencia en la educación superior europea, elaborar puntos de referencia para el análisis y la armonización de los requisitos de la titulación de la/el enfermera/o e incentivar a las universidades a desarrollar estrategias de autorregulación, definir su impronta mediante la delimitación de las competencias generales que caracterizarán a sus egresados y que cada grupo disciplinar defina las competencias específicas, es decir, utilizan el proyecto como herramienta para que las universidades mejoren la calidad de la educación $(1,2)$. América Latina se incorpora al Proyecto Tuning en el año 2004 declarando que "la Universidad como actor social tiene desafíos y responsabilidades, independientemente del lugar del hemisferio en el cual se encuentre" (3).

Otro de los aspectos a considerar en la formación de profesionales de enfermería, son las reformas del sector salud. No sólo Chile sino todos los países de la región de las Américas requieren contar con sistemas de salud equitativos, eficientes, efectivos, sustentables y orientados hacia la satisfacción de las necesidades de salud de las poblaciones. Resulta entonces fundamental, para lograr los objetivos propuestos, contar con "recurso humano", capacitado, con saberes actualizados y competente, donde el profesional de enfermería tiene un rol preponderante. Es importante considerar, en las competencias de egreso de este profesional, los cambios que se han producido en aspectos demográficos, en donde nuestro país está en franco envejecimiento y con expectativa de 
vida similar a países desarrollados, con perfiles epidemiológicos propios de estos países, pero también coexisten patologías de países en desarrollo que obligan a una formación que responda a estos nuevos requerimientos. Es fundamental incorporar, además, el entorno social, político, legal, cultural y económico en que se encuentra nuestro país, puesto que estas variables deben ser consideradas en los procesos educativos que van a guiar la formación de enfermeras/os, en la calidad de las prestaciones de salud y en la entrega de un cuidado experto otorgado a la población, situación que exige la incorporación de nuevas competencias de los profesionales que allí laboran, las que deben estar en concordancia con las competencias de egreso.

Los actuales currículos y perfiles profesionales están en proceso de cambio, así en algunas instituciones se ha incorporado metodologías centradas en el estudiante, se están realizando esfuerzos por implementar un sistema justo de equivalencias de estudios expresado en el sistema de créditos transferibles, definido como el tiempo necesario que requiere el estudiante para lograr un aprendizaje (4), que además favorezca el intercambio estudiantil al tener planes de estudios comparables y homologables.

El proceso de enseñanza-aprendizaje, en los últimos años ha experimentado cambios, desde un modelo convencional, centrado en el profesor, se han realizado modificaciones de forma, cuyas nuevas didácticas y estrategias se han focalizado en el estudiante, incidiendo directa y notoriamente en el grado y en la calidad del aprendizaje alcanzado por el educando, apreciándose una mayor motivación por aprender, oportunidades para el desarrollo de procesos mentales superiores, mayor autonomía en la toma de decisiones, entre otras.

Considerando a las universidades en este análisis, se podría decir que el tema de la calidad de la formación, actualmente, se encuentra en desarrollo y asociado a los procesos de acreditación, aplicados por la Comisión Na- cional de Acreditación (CNA) (5), tanto en el ámbito institucional como en las carreras que en ellas se imparten, de manera que estas instituciones den garantía pública de la calidad de la formación de profesionales y de los servicios ofrecidos.

Por lo expuesto precedentemente es que surge un proyecto de renovación curricular en red, de cuatro instituciones estatales, regionales, comprometidas con la educación superior que imparten la carrera de enfermería en el norte del país, a saber, las Universidades de Tarapacá, Arturo Prat, de Antofagasta y de La Serena, quienes se proponen intervenir, sustancialmente, en los problemas derivados de sus actuales currículos, de sus metodologías y prácticas docentes. Al mismo tiempo, pretende optimizar la gestión académica, asegurando con ello la calidad y pertinencia de la formación profesional de enfermería (6).

Para lograr lo señalado anteriormente se proponen como líneas de acción: la evaluación de los actuales currículos según estándares propuestos por la Comisión Nacional de Acreditación; el diseño de un nuevo currículum basado en competencias, centrado en el estudiante como persona; el desarrollo de un sistema de créditos transferibles, homologables y acumulables; el perfeccionamiento docente; el fortalecimiento y desarrollo de la red de enfermería y, finalmente, el establecimiento de vínculos de cooperación con entidades nacionales e internacionales que faciliten la movilidad estudiantil y académica, con su respectivo reconocimiento y certificación.

\section{MARCO REFERENCIAL}

La formación por competencias está probando ser una herramienta válida para la concreción de lo que el informe Délors plantea como los cuatro pilares del aprendizaje del siglo XXI: conocer y aprender a aprender, saber hacer, saber ser y saber vivir en paz con 
los demás (cultura de paz). La naturaleza integral de las competencias permite concretar, aunque sea en una forma inicial, la aspiración de ofrecer una educación que facilite los desarrollos mencionados, como a su vez la holicidad (7).

Las competencias se desarrollan a través de diversas y variadas experiencias de aprendizaje en cuyo campo de conocimiento se integran tres tipos de saberes: conceptual (saber conocer) el que utiliza procesos tales como atención, adquisición, recuperación, trasferencia y evaluación. Los instrumentos cognitivos son las nociones, proposiciones, conceptos, y categorías. Las estrategias utilizadas son las cognitivas y las metacognitivas. En lo procedimental (saber hacer) el proceso es de desempeño. Los instrumentos utilizados son actuación, procedimientos y técnicas. Las estrategias son actuacionales. En lo concerniente a lo actitudinal (saber ser) los procesos son sensibilización, personalización y cooperación. Los instrumentos utilizados son afectivos, valores, actitudes y normas. Las estrategias son emocionales sociales y motivacionales.

Todos los anteriores son aprendizajes integradores que involucran la reflexión sobre el propio proceso de aprendizaje (metacognición) y la movilización de saberes $(8,9)$.

La formación de profesionales competentes siempre ha sido la razón de ser de la educación terciaria, sin embargo hoy surge con más fuerza el ser competente. Así se ha establecido una diferencia entre las competencias del mundo técnico y el mundo profesional $(10,11)$ que nos entregan algunas pistas. "Ser competente es saber hacer y saber actuar entendiendo lo que se hace, comprendiendo cómo se actúa, asumiendo de manera responsable las implicancias y consecuencias de las acciones realizadas y transformando los contextos a favor del bienestar humano" $(12,13)$.

Es del todo necesario favorecer, en los futuros profesionales, el ejercicio profesional en un mundo en el que priman la com- plejidad, los cambios constantes, en síntesis deben "aprender a navegar en un océano de incertidumbres, a través de archipiélagos de certeza" (14).

En conclusión, el proyecto de renovación curricular propuesto por la Red de Enfermería de la Macrozona Norte del país busca producir transformaciones reinterpretando las prácticas que constituyen el estilo histórico de la disciplina, pasando desde:

- Estudiantes que adquieren información, a estudiantes autónomos con capacidad de emprendimiento que se insertan exitosamente en el mundo global.

- Profesores que transmiten información, a educadores mediadores entre el conocimiento existente y el aprendiz y el medio, construyendo comunidades de aprendizajes, permitiéndose de este modo el desarrollo de procesos mentales superiores, donde el estudiante construye su propio aprendizaje, convirtiéndose en un aprendizaje significativo. Donde las estrategias metodológicas planteadas y las experiencias de aprendizajes confluirán en el desarrollo de competencias $(15,16)$.

- Programas de estudio rígidos basados en la acumulación de conocimiento a currículos flexibles, orientados a desarrollar competencias para el desempeño personal y profesional globalizado.

- Formación parcializada, fragmentada y orientada sólo a su profesión, a una formación integral que incorpore tanto las competencias propias de su especialidad como el desarrollo de habilidades que les permita insertarse a un mundo laboral cada día más exigente, competitivo y demandante.

- Enseñanza tradicional y homogénea a un proceso de aprendizajes significativos, considerando el contexto, la historicidad del estudiante y del profesor, desde donde emerjan nuevos saberes acorde a principios y valores universitarios.

- Relación de enseñanza individualista y 
directiva a un aprendizaje colaborativo y participativo, donde profesores, estudiantes y otros agentes contribuyen interactiva y solidariamente $(17,18)$.

\section{ELABORACIÓN DEL DISEÑO CURRICULAR}

Para dar cumplimiento a los objetivos planteados en el proyecto se establecieron convenios de colaboración entre las cuatro instituciones involucradas, Universidad de Tarapacá, Universidad Arturo Prat, Universidad de Antofagasta y la Universidad de La Serena. Se revisó, ampliamente, la literatura y tendencias curriculares, se asistió a múltiples talleres y reuniones convocados por el Ministerio de Educación, se estableció una organización en las unidades participantes para asegurar la contribución de académicos, estudiantes, egresados y empleadores. Se contrató una asistencia técnica que colaboró en el esbozo y definición de los elementos constitutivos del marco filosófico y su interrelación con los conceptos centrales presentes en el currículo, dando como resultado el marco contextual, conceptual, filosófico, educativo, valórico y disciplinar en el cual se inserta el currículo. A su vez se contó con la asesoría de expertos, lo que permitió adentrarnos en la disciplina de enfermería, analizar en profundidad el cuidado como centro del accionar del profesional enfermera/o, éste cuidar - cuidado cuidando y ser en todo momento presencia verdadera y una ayuda restauradora durante el proceso de salud, en cualquier estado del ciclo vital, objeto de estudio y desarrollo de la disciplina de enfermería.

Uno de los aspectos más relevantes de este nuevo diseño curricular es la construcción del perfil profesional que se confeccionó considerando las recomendaciones dadas por los expertos, por el Proyecto Tuning Latinoamericano, y los fundamentos dados por la Comisión Nacional de Acreditación, vali- dándose el perfil mediante consultas con expertos nacionales e internacionales, en talleres con empleadores, egresados y estudiantes de las instituciones involucradas en la red.

Junto a la elaboración del perfil profesional, se definieron las competencias de egreso, considerando tanto las genéricas como las específicas que van a caracterizar a los profesionales titulados de las escuelas de enfermería de la red norte. En función de éstos, se establecieron los principales dominios que se abordarán en el currículo, que deberían estar presentes de acuerdo a las tendencias en la praxis y disciplina de enfermería de la Red Norte, las que fueron definidas como sigue; proveedor de cuidados, investigación y emprendimiento, promoción de la salud y prevención de la enfermedad, administración y gestión del cuidado. También se analizaron y ejercitaron diversas metodologías de aprendizajes centradas en el estudiante, que permita desarrollar competencias.

Todos estos elementos sirvieron de base para elaborar la matriz de competencias por niveles de formación, de acuerdo al perfil de egreso definido y los cuatro dominios establecidos. En esta matriz de competencias se insertaron los módulos de aprendizajes, a saber: doce módulos comunes, considerando afinidad en los aprendizajes a entregar, el tiempo que ocupara el estudiante en lograr dichos aprendizajes, siendo los módulos más relevantes, los siguientes: ser humano, familia y comunidad como centro del cuidado, enfermería como ciencia del cuidado, cuidado en el desarrollo humano y salud familiar, gestión y gerencia del cuidado, comunicación e interacción en el cuidado, entre otros. Además se definieron ocho módulos, dos por cada unidad académica, de acuerdo a las fortalezas de cada una y la incorporación de las competencias genéricas, definidas por cada universidad, las que darán la impronta institucional.

Para concretar este trabajo se realizaron diversas actividades, destacándose, entre ellas, pasantías con objetivos específicos a 
universidades con trayectoria en la implementación de los currículos basados en competencias, de las cuales podemos destacar la Universidad de Zaragoza, la Internacional de Catalunya, Europea de Madrid, de Sevilla, de España, Universidad de Nueva León de México, Universidad de Antioquia de Colombia, entre otras. Estas pasantías permitieron conocer los currículos, las metodologías innovadoras y participativas utilizadas, la implementación del modelo curricular, el trabajo en aula, entrevistas estructuradas con los directivos de las diversas escuelas. Otro motivo de interés fue el conocimiento del sistema de bibliotecas y los textos de estudio, el uso de tecnologías e instrumentos para lograr aprendizajes, conocimiento del sistema de evaluación centrada en el estudiante y seguimiento curricular.

Se recogió la experiencia del diseño, las estrategias que establecieron para su implementación, las principales dificultades y debilidades encontradas en los nuevos procesos que implica la formación por competencias.

Se contó con expertos en educación para la elaboración de las asignaturas que permiten plasmar los distintos saberes y disciplinas, dando un enfoque sistémico e integrado al currículo.

Se elaboró un perfil consensuado entre las universidades de la red con los distintos actores que intervienen en el proceso de enseñanza-aprendizaje y las competencias de egreso de un profesional enfermera/o generalista, Licenciado en Ciencias de la Enfermería, que sea capaz de dar respuesta no sólo a un mundo laboral específico, como es el de Enfermería, sino también la de un profesional inserto en un mundo dinámico, cambiante, con sensibilidad social, claros principios éticos en el cual se centra el cuidado humano, teniendo como marco de referencia las competencias genéricas y específicas emanadas del proyecto Tuning América Latina, las competencias definidas por el Comité Técnico de Enfermería de la Comisión Nacional de Acreditación, y diversas miradas internacionales.
Un currículo flexible, que permita el intercambio estudiantil y académico entre las instituciones socias, reconociendo las fortalezas y los acentos de las instituciones involucradas, un currículo que coloca al estudiante en el centro del proceso enseñanza aprendizaje, basado en la reflexión, el análisis, en la construcción de su aprendizaje. Considerando que éste debe tener un tenor significativo, la historicidad, el contexto, la reflexión sobre la práctica, atendiendo que el aprendizaje tiene el carácter de un proceso inherente al ser humano, permanente, transformador, conducente al desarrollo del potencial humano.

Considera principios de mediación para el proceso de enseñar-aprender, se entiende como una interacción basada en una experiencia entre el mediador y el aprendiz, donde lo esencial es la construcción conjunta del desarrollo del potencial humano, en el cual se inserta un sistema de evaluación, con carácter, formativo, permanente, constructivo, retroalimentador, basada en la reflexión, autocrítica, conducente al desarrollo del proceso educativo. En función del proceso de aprendizaje contribuyendo a la retroalimentación del proceso de enseñanza. Incorpora diversos aspectos del proceso educativo y diversifica los tipos de evaluación, que conduce a la toma de decisiones.

Se ha establecido en función del perfil de egreso una matriz de subcompetencias por cada nivel de la formación, identificando cada uno de los saberes inherentes a la competencia de egreso, lo que permite dar cuenta de logros de aprendizajes en los diferentes saberes para la consecución del perfil de egreso.

Las asignaturas son el corolario del trabajo desarrollado, dejando explícita la integración, donde quedan claramente establecidos las competencias a desarrollar, la fusión de los saberes, en virtud de los logros de aprendizajes, estableciendo las metodologías, las actividades de aprendizaje y la evaluación de los resultados de aprendizaje, en función de 
las competencias que se desean desarrollar, con un mirada sistémica.

\section{CONSIDERACIONES FINALES}

Trabajar un currículo basado en competencias es una innovación en la formación, un cambio paradigmático donde converger aspectos; ontológicos epistemológicos y metodológicos. Implica un replanteamiento de todos los aspectos curriculares, los ejes centrales de la formación, las metodologías utilizadas, las actividades de aprendizaje, donde el estudiante es el centro del proceso y se debe responsabilizar de este. El gran objetivo es lograr resultados de aprendizajes significativos, lo que obliga a remirar las formas de evaluar, y es sin duda una oportunidad de hacer cambios trascendentales y profundos en educación superior $(19,20)$.

La experiencia de trabajar un currículo en red ha sido un hermoso y valiosísimo aprendizaje, en primer término por la oportunidad de constituir un grupo de reflexión académico, que anhelan aportar a la formación de enfermería, colocando la expertiz, creatividad y sabiduría de cada uno de los participantes en los diversos encuentros para analizar documentos; estudiar la tendencia actual en construcción curricular; dialogar tanto al interior de las unidades como interunidades, acerca de la formación de un futuro profesional, aunar miradas y vincularnos con el sector laboral, analizando las necesidades actuales de las competencias requeridas para enfrentar un mundo laboral desafiante; y finalmente conocer y vincularnos internacionalmente con académicos/as que también están pensando y recreando la formación de profesionales provenientes de universidades de gran prestigio y reconocidas por sus pares.

Es gratificante realizar el aporte concreto en educación en enfermería de un nuevo perfil, con nuevos desafíos y grandes responsabilidades que se le asignan a la/el enfermera/o, con una nueva mirada de lo que es hoy este profesional y apoyar su actuar en los fundamentos de la disciplina de enfermería, sin dejar de considerar todas las variables sociodemográficas, epidemiológicas, culturales, que van a influir poderosamente en la formación. En un mundo sorprendido e impactado por cambios y tecnologías, que nos deslumbran y a la vez desconciertan, sigue plenamente vigente la necesidad del cuidado profesional de las personas, familias y comunidad en el cual se incorporan las nuevas herramientas tecnológicas, que sin duda son un gran aporte, más sin perder nunca la característica esencial de este arte de cuidar que es propio de Enfermería, esta es la humanidad, la sensibilidad, el respeto por el otro, este es el gran desafío que se debe imprimir como un sello a todas las futuras generaciones de enfermeras/os y este es uno de los desafíos de esta red nortina, que contribuye desde el calor del desierto al futuro de la enfermería chilena.

\section{REFERENCIAS}

1. González J., Wagemaan R (editores). Tuning Educational Structure in Europe, Informe Final. España: U. de Deusto; 2003.

2. Martínez R, Cibanal J. Enfermería Integrada. Una aproximación docente al Espacio Europeo de Educación Superior (EEES). España: Universidad de Alicante; 2005.

3. Benietone P, Esquetini C, González J, Marty M, Sinfi G. Wagenaar R (editores). Reflexiones y proyecciones de la Educación en América Latina. Informe final Proyecto Tuning América Latina 2004-2007. [Internet]; 2007 [citado mayo 2010]. Disponible en: http://tuning.unideusto.org/tuningal

4. Guía Práctica para la instalación del SCT - Chile [Internet]; 2007 [citado mayo 2010]. Disponible en: www.sct-chile.cl 
5. Comisión Nacional de Acreditación Internet. Chile. Perfil Profesional Mínimo de la Carrera de Enfermería. [Internet]; 2001 [citado mayo 2010]. Disponible en: http://www.cnachile.cl

6. Delors J, Al Mufti I. La educación encierra un tesoro: informe a la UNESCO de la Comisión Internacional sobre la Educación para el siglo XXI. Madrid: Santillana. [Internet]; 1996 [citado mayo 2010]. Disponible en: http://www. unesco.org/education /pdf/

7. Tobón S. Formación Basada en Competencias 2a. ed Bogotá: ECOE; 2006.

8. Posada R. Formación superior basada en competencias, interdisciplinariedad y trabajo autónomo del estudiante. $R e$ vista Iberoamericana de Educación [Internet]. 2004 [citado 8 enero 2010]:1-34. Disponible en http://www.rieoei.org/ deloslectores/648Posada.PDF

9. Pinto Cueto L. Currículo por competencias: necesidad de una nueva escuela. Revista de Educación y Cultura. 1999; 43: 10-17.

10. Perrenoud P. Construir Competencias, ¿Es darle la espalda a los saberes? Red U. Revista de Docencia Universitaria, 2008, número monografías II. Disponible en www.redu. um.es/Red_Um2

11. Hernández D. Políticas de certificación de competencias en América Latina. CINTERFOR/OIT. Seminario: La Nueva Educación Profesional. Sao Paulo; 2000.

12. Montenegro I. Aprendizaje y Desarrollo de las Competencias, Colombia: Magisterio; 2003.
13. Kaluf C. Reflexiones sobre Competencias y Educación. Ponencia presentada a Seminario técnico internacional "Evaluación de competencias de egresados universitarios". Santiago, Chile: CINDA; 2003.

14. Irigoyen M, Varas F. Competencias laborales. Un modelo de competencias para el sector salud. Programa de desarrollo de recurso humano. División de sistema y servicios de salud OPS /OMS-OIT. Montevideo; 2002.

15. Grinberg M, Morin E. El pensamiento complejo. 2a. ed. Madrid: Campo de Ideas; 2002.

16. Bozu Z, Canto Herrera J. El profesorado universitario en la sociedad del conocimiento: competencias profesionales docentes. Revista de formación e Innovación Educativa Universitaria. 2009; 2 (2): 87-97.

17. González M. Diseño, implementación y evaluación de programas por Competencias Profesionales Integradas. México: Universidad de Guadalajara; 2002.

18. Perrenoud P. Diez nuevas competencias para enseñar. 5a ed. Barcelona: Biblioteca de aula; 2007.

19. Díaz Barriga F, Hernández G. Estrategias docentes para un aprendizaje significativo: una interpretación constructivista. 2a. ed. México D.F.: Mc Graw-Hill; 1999.

20. Irigoyen JJ. Jiménez MY, Acuña KF. Competencias en educación superior. RMIE [Internet]. 2011 [citado mayo 2011]; 16 (48): 243-266. Disponible en http:// www.scielo.org.mx/pdf/rmie/v16n48/ v16n48a11.pdf 